\title{
Efeitos de borda sobre a comunidade de pteridófitas na Mata Atlântica da região de Una, sul da Bahia, Brasil ${ }^{1}$
}

\author{
MATEUS L.B. PACIENCIA ${ }^{2,4}$ e JEFFERSON PRADO ${ }^{3}$
}

(recebido: 28 de agosto de 2003; aceito: 1 de julho de 2004)

\begin{abstract}
Edge effects on pteridophyte community in Atlantic rain forest of the Una region, South of Bahia State, Brazil). Edge effects are one of the most important consequences of forest fragmentation. This paper concerns how richness and abundance of pteridophytes vary when forest interiors and edges are compared on the fragmented Una landscape, state of Bahia. All ferns found up to $1 \mathrm{~m}$ above the ground were collected on 36 parcels of $120 \times 10 \mathrm{~m}$. Six sampling units were established on continuous forest areas ( $>900 \mathrm{ha}$ ) and six more on forest fragments ( $\leq 100 \mathrm{ha}$ ). These units were allocated within three sampling blocks of $5 \times 5 \mathrm{~km}$ over Una landscape. Each area had its parcels positioned at $20 \mathrm{~m}, 40 \mathrm{~m}$, and further than $100 \mathrm{~m}$ from the border line, which was drawn by the cattle pastures areas passing through forest patches on the landscape. There was no significant variation of abundance among studied units, although richness decreases in all blocks, when parcels are closer to the matrix. Species richness is reestablished 20-30 m far from the border, where it has no significant difference to that of forest interior. In addition interior and border of forests are totally different environments, regarding pteridophytes community. Forest interior communities are almost not affected by edge effects. Therefore, border effects have a short penetration on pteridophytes communities, although a large scale raise on the amount of borders might result on the extinction of some local forest species.
\end{abstract}

Key words - Bahia, edge effect, ferns, forest fragmentation

RESUMO - (Efeitos de borda sobre a comunidade de pteridófitas na Mata Atlântica da região de Una, sul da Bahia, Brasil). Uma das consequiências da fragmentação de hábitats florestais melhor estudadas até o momento são os chamados efeitos de borda. Neste estudo, foi verificado como variam a riqueza e a abundância das pteridófitas quando são comparados interiores e bordas de áreas florestais, em Una, BA. Foram inventariadas todas as pteridófitas a até $1 \mathrm{~m}$ do solo, em 36 parcelas de $120 \times 10 \mathrm{~m}$ ( 0,12 ha), estabelecidas em áreas de mata contínua (> 900 ha) e fragmentos de mata ( $\leq 100$ ha), distribuídas na região entre três blocos amostrais de $5 \times 5 \mathrm{~km}$. Em cada uma das áreas, locaram-se as parcelas a 20, 40 e a mais de $100 \mathrm{~m}$ da divisa da mata com relação às áreas adjacentes. Os resultados mostram que não há variação na abundância entre as unidades consideradas. No entanto, a riqueza de samambaias é diferente entre blocos amostrais, indicando que as florestas de Una podem ser heterogêneas quanto aos aspectos ambientais. Além disso, a riqueza decai nas parcelas de borda mais próximas à matriz, em todos os blocos; a partir de uma distância aproximada de 20-30 m a riqueza é restabelecida, tornando-se similar à do interior da floresta. Também fica claro que os interiores de floresta constituem ambientes totalmente distintos daqueles de borda, no que se refere à comunidade de pteridófitas, de modo que, praticamente, não são afetados pelos efeitos de borda. Pôde-se concluir que os efeitos de borda sobre as pteridófitas penetram muito pouco no interior da floresta, mas deve-se ressaltar que a formação de novas bordas em larga escala pode vir a extinguir espécies florestais da região.

Palavras-chave - Bahia, efeito de borda, fragmentação florestal, samambaias

\section{Introdução}

A substituição de grandes áreas de florestas por ecossistemas diferentes leva à criação de fragmentos florestais isolados, imersos em uma matriz de ambientes não florestais ou "matriz inter-hábitat" (Forman \&

1. Parte da dissertação de mestrado do primeiro autor, Universidade de São Paulo.

2. Universidade Paulista, Herbário UNIP, Av. Paulista 900 $1^{\circ}$ andar, Bela Vista, 01310-100 São Paulo, SP, Brasil.

3. Instituto de Botânica, Caixa Postal 4005, 01061-970 São Paulo, SP, Brasil.

4. Autor para correspondência: matinas@unip.br
Godron 1986, Franklin 1993). O aumento da área de contato das florestas com a matriz decorrente do isolamento das manchas florestais (Williams-Linera et al. 1997) promove uma alteração no movimento energético, material e no fluxo de organismos entre tais ambientes (Wiens et al. 1993). De maneira geral, estas modificações nas áreas mais externas dos fragmentos florestais, i.e. bordas do fragmento, geradas pelo contato com a matriz, são chamadas "efeitos de borda" (Murcia 1995).

Nas bordas de fragmentos florestais, comunidades vegetais da matriz e da floresta se encontram e, assim, as bordas podem ser entendidas como áreas de transição entre unidades da paisagem de hábitat e não-hábitat, 
regidas pelas "forças de interação" entre as mesmas (Holland 1988 apud Metzger 1999).

Segundo alguns estudos, as bordas são áreas mais expostas às perturbações externas, com maior diversidade de espécies vegetais (decorrente da sobreposição de espécies do interior e da matriz), maior cobertura e densidade de indivíduos e maior produtividade primária (Didhan \& Lawton 1999), sendo esta última característica percebida devido ao aumento da taxa fotossintética total nestes ambientes (MacDougall \& Kellman 1992).

Em suma, funcionalmente, bordas são áreas onde a intensidade dos fluxos biológicos entre as unidades de paisagem se modifica de forma abrupta, devido à mudança abiótica repentina das matrizes para os fragmentos e vice-versa (Metzger 1999). Vista do interior da mata, tal mudança pode ser evidenciada por um aumento da penetração da luz solar (Murcia 1995) e maior incidência de ventos (Laurance et al. 1998a). Estas alterações podem ocasionar a elevação da temperatura no ambiente (Nichol 1994) e o conseqüente aumento da evapotranspiração (Matlack 1993), proporcionando, assim, a diminuição da umidade relativa do solo e do ar (Kapos 1989), o que favoreceria o estabelecimento de estresse hídrico (Esseen \& Renhorn 1998).

Em última análise, todo este processo acaba sendo um fator selecionador das comunidades capazes de se instalar e utilizar as bordas como área de desenvolvimento, devido às adaptações necessárias para as espécies habitarem com sucesso esses ambientes ecotonais (Willson \& Crome 1989, Malcolm 1994).

Não obstante, parece não haver um padrão muito claro sobre até que ponto as alterações micro-climáticas das bordas podem ser percebidas no interior dos fragmentos. Ao estudar fragmentos em reservas florestais próximas a Manaus, AM, Kapos (1989) mostrou que a distância de penetração destas alterações chega a $40 \mathrm{~m}$, a partir do limite físico do fragmento com a matriz. Outros autores relatam penetrações de efeitos menos intensas, como 15-25 m (Williams-Linera 1990), 7-12 m (MacDougall \& Kellman 1992), 10-20 m (Esseen \& Renhorn 1998) e atentam que as alterações estão intimamente associadas à idade da borda e ao tempo de observação (coleta de dados) (Kapos et al. 1997).

Embora existam dados algo conflitantes a este respeito, é consenso entre os pesquisadores que fragmentos florestais muito pequenos podem vir a ser, no seu todo, extensas bordas de florestas e, então, as alterações podem manifestar-se multiplicativamente, pois o micro-clima da floresta é fortemente influenciado pela distância em relação à periferia do fragmento.

Apesar das bordas de fragmentos poderem constituir ambientes inóspitos para algumas espécies florestais, e possivelmente para a maioria delas, dadas a redução da área de utilização e as alterações da forma e das características estruturais do fragmento, esses ecótonos não devem ser vistos necessariamente como localidades adversas ao estabelecimento e ao desenvolvimento das espécies florestais. Isto porque os grupos biológicos nem sempre respondem da mesma maneira aos efeitos de borda e esses, por sua vez, não devem se manifestar de maneira exatamente igual em todas as bordas.

Segundo Murcia (1995), os efeitos de borda aplicam-se diferentemente sobre guildas ou grupos biológicos distintos, qualitativa (tipo de efeito) e quantitativamente (intensidade). Por exemplo, enquanto se pode observar, próximo à borda, um incremento da mortalidade das espécies arbóreas florestais de grande porte (DAP > $20 \mathrm{~cm}$, ver Laurance 1991), ao mesmo tempo, verifica-se que há um aumento das espécies pioneiras e tolerantes às condições climáticas do ambiente alterado (Williams-Linera 1990, Laurance et al. 1998b).

Entretanto, a maioria dos estudos de borda referentes à vegetação, enfocaram os efeitos produzidos sobre comunidades de angiospermas arbóreas e arbustivas (Williams-Linera 1990, Laurance 1991, Matlack 1993, Fox et al. 1997, Laurance et al. 1998a); poucos se dedicaram a estudar espécies herbáceas (Matlack 1994), ou outros grupos taxonômicos como líquens fruticosos (Esseen \& Renhorn 1998) e samambaias arbóreas (Bernabe et al. 1999). Ainda é necessário estudar a resposta de um maior número de grupos frente aos efeitos de borda, principalmente nas florestas tropicais, uma vez que o grande emblema atual desses ecossistemas é o extenso depauperamento e a redução de suas áreas originais (Turner 1996).

Em estudo recente, Paciencia \& Prado (dados não publicados), procuraram estudar as respostas das samambaias florestais frente aos aspectos gerais da fragmentação. No presente trabalho, estudou-se a forma pela qual as pteridófitas respondem à introdução das bordas de florestas, em fragmentos que outrora eram interligados e, portanto, possuíam extensas áreas de interiores florestais, na Mata Atlântica da região de Una, BA. Para tanto, foi verificado, especificamente, se existe variação da riqueza de espécies e da abundância dos indivíduos nas áreas de borda, procurando-se definir qual é o limite de penetração do efeito de borda para a comunidade de pteridófitas como um todo, uma vez que, 
estimar a escala do efeito de borda é essencial para a preservação da flora de florestas em regiões afetadas pela fragmentação. Estas duas variáveis biológicas são os principais tipos de efeitos de borda diretos estudados até o momento, pois determinam a distribuição das espécies, e suas mudanças estão provavelmente relacionadas às condições físicas nas proximidades das bordas.

\section{Material e métodos}

Caracterização da área de estudo - A área de estudo situa-se na floresta ombrófila densa de baixada do estado da Bahia, adjacente à Reserva Biológica de Una (Rebio Una - $15^{\circ} 10^{\prime} \mathrm{S} \mathrm{e}$ $39^{\circ} 03^{\prime} \mathrm{W}$ ), município de Una, BA (figura 1). A região é dominada por um clima do tipo Af (Koeppen 1948) 3-4 quente e úmido sem estação seca e com precipitação anual superior a $1.300 \mathrm{~mm} 3-4$, condicionante de floresta tropical (Gouvêa 1969). As chuvas são concentradas nos meses de janeiro a abril, outubro a dezembro e no mês de agosto e as médias anuais de temperatura ficam próximas a $24{ }^{\circ} \mathrm{C}$, variando entre $26^{\circ} \mathrm{C}$ no verão e $18^{\circ} \mathrm{C}$ no inverno (SEI 1998).

A vegetação original da região é denominada Mata Higrófila Sul-Baiana (Gouvêa et al. 1976), uma formação de floresta ombrófila que predomina, em geral, junto aos cordões litorâneos e zonas de baixas altitudes no sul e sudeste da Bahia. Toda a região de Una dominada pela Mata Higrófila Sul-Baiana é considerada uma área prioritária para a conservação biológica mundial (CI 2002), dadas as elevadas taxas de endemismo que apresenta, para diversos grupos biológicos, tanto da flora (Prance 1982, Mori et al. 1983, Thomas et al. 1998) quanto da fauna (Haffer 1974, Rylands 1982, Brown 1991).

Contudo, a floresta ombrófila encontra-se reduzida em termos de sua área total (Alger 1997), sendo que apenas cerca

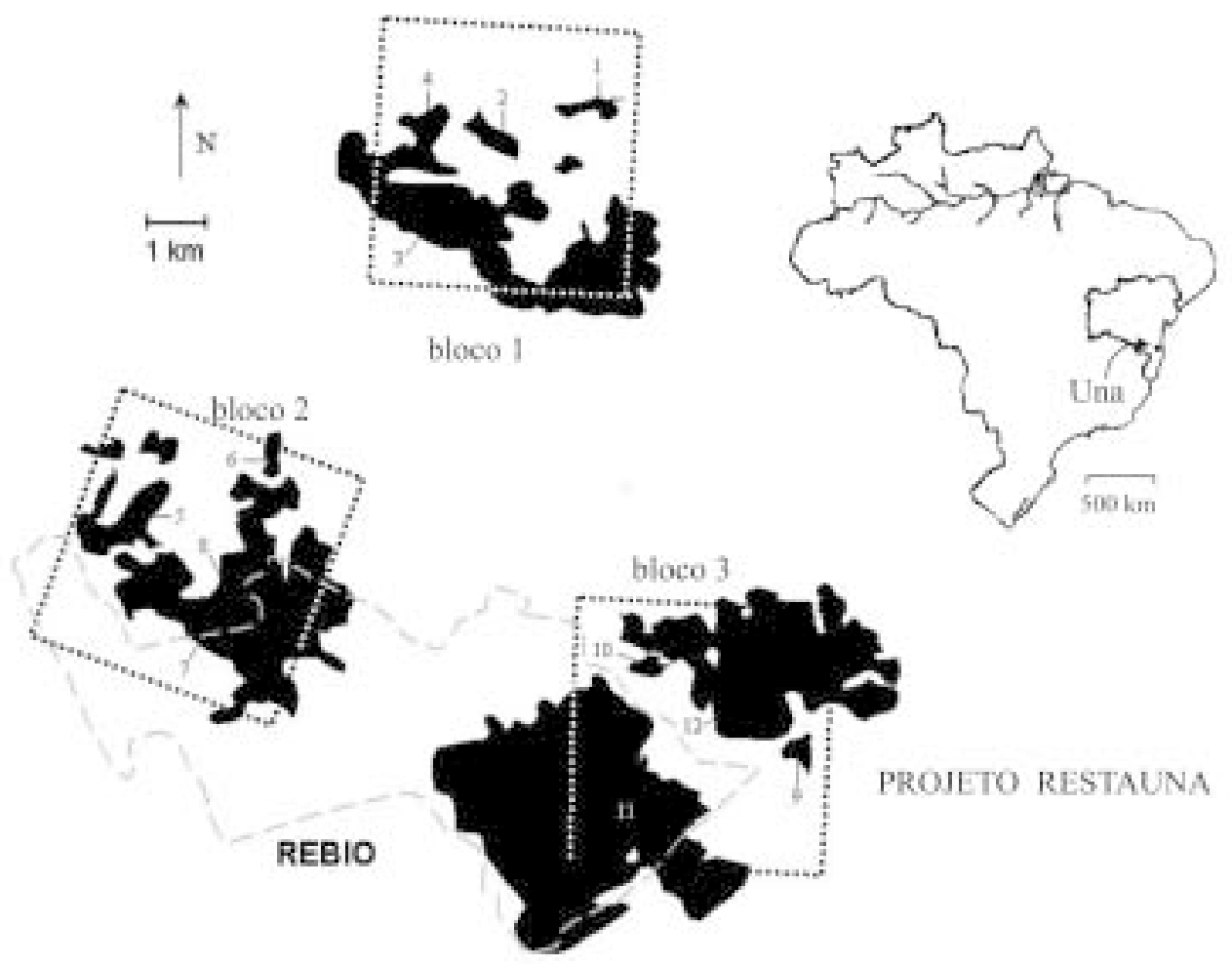

Figura 1. Área de estudo na região de Una, sul da Bahia, Brasil, com os três blocos experimentais de $5 \times 5$ km e os sítios (UA) nos quais foram conduzidas as amostragens. Linhas pontilhadas representam os limites do blocos; linhas tracejadas mostram os limites da Rebio Una; áreas negras representam a floresta ombrófila densa e áreas brancas a matriz da paisagem. Os sítios estudados foram: 1= Fazenda do Mário, 2 = Fazenda da Cachoeira Lisa, 3 = Controle do Rumo, $4=$ Fragmento da Braúna, 5 = Fragmento do Jupará, 6 = Fazenda do Dr. Amilton, 7 = Rebio, 8 = Fazenda do Coco, 9 = Fragmento do Pé no Chão, 10 = Fragmento da Juerana, 11 = Trilha do Príncipe (sede da Rebio), 12 = "Canopy Walking".

Figure 1. Study area in Una region, South of Bahia, Brazil, showing three experimental blocks $(5 \times 5 \mathrm{~km})$ and its inventoried sites (UA). Stippled lines represent the blocks, and dashed lines are the legal Una Reserve limits. Shaded areas inside the blocks represent the ombrophilous forest, and the unshaded ones are the landscape matrix. The inventoried sites were: $1=$ Mário's farm, 2 = Slide falls farm, 3 = Rumo's control, 4 = Braúna's fragment; 5 = Jupará's fragment, $6=$ Dr. Amilton's farm, $7=$ Una Biological Reserve (Rebio), $8=$ Coconut's farm, $9=$ Firm land fragment, $10=$ Juerana's fragment, $11=$ Prince track $($ Rebio's headquarter place), 12 = Canopy Walking. 
de 30.000 ha da região devem encontrar-se cobertos por floresta úmida, representando aproximadamente $10 \%$ do total original. Não obstante, grande parte do montante remanescente está fragmentado e submetido a intensa pressão antrópica, devido à retirada de madeira, ao desflorestamento generalizado para a implementação das atividades agropecuárias e à caça ilegal. Assim, verifica-se, em Una, um mosaico ambiental formando a paisagem local, de feições reticulares, onde os remanescentes florestais inserem-se em uma matriz profundamente heterogênea, composta por pastagens e cultivos agrícolas diversos.

Desenho experimental - O presente estudo foi desenvolvido como parte integrante do Projeto Resta Una - Remanescentes de Floresta da Região de Una, sul da Bahia (Nema/Uesc http//www.restauna.org.br), que visou efetuar comparações entre as respostas de diversos grupos biológicos frente ao processo da fragmentação de hábitats florestais em Una.

O trabalho de campo foi conduzido nas principais áreas de vegetação nativa remanescente da região de Una, BA, em 36 parcelas de 0,12 ha $(120 \times 10 \mathrm{~m})$ (figura 1$)$, verificando-se qual é o poder de penetração do efeito de borda sobre assembléias de pteridófitas florestais.

Foi investigado como variam a riqueza de espécies e a abundância de indivíduos, ao longo de um gradiente de distância, fragmento adentro, a partir da linha de borda adjacente à matriz da paisagem. As parcelas, ou unidades amostrais (UA) foram distribuídas em três blocos de amostragem, de $5 \times 5 \mathrm{~km}$, dispostos na região de acordo com a presença das maiores áreas de floresta existentes, de modo que cada bloco apresentasse um mesmo número de UA, sempre contando com dois fragmentos de mata (áreas com 25-100 ha) e uma área de mata contínua (área-core, maior que 900 ha).

As 36 unidades amostrais foram estabelecidas em três pontos dentro dos fragmentos e das matas contínuas, a diferentes distâncias a partir do limite dos mesmos com a matriz constituída por pastagens. A primeira parcela foi estabelecida a até $20 \mathrm{~m}$ da porção limítrofe do fragmento em relação à matriz, a segunda parcela, a $20 \mathrm{~m}$ da primeira, e a terceira, a pelo menos $100 \mathrm{~m}$ da linha divisória da borda.

Dessa forma, caracterizaram-se três tipos de unidades amostrais distintas: BA - parcela de amostragem cuja porção central foi disposta paralelamente à linha de borda, a até $20 \mathrm{~m}$ fragmento adentro; $\mathrm{BB}$ - parcelas implantadas a até $40 \mathrm{~m}$ da linha de borda (a $20 \mathrm{~m}$ de distância de BA); I - parcelas implantadas a mais de $100 \mathrm{~m}$, fragmento adentro, caracterizando as unidades de interior de mata. Independentemente da distância exata das primeiras parcelas (BA) em relação à linha de borda, as parcelas intermediárias (BB) sempre distaram $20 \mathrm{~m}$ destas.

O desenho experimental permitiu uma análise dos dados coletados em "mesoescala" (entre os diferentes tipos de UA) e também em "macroescala" (entre blocos), sendo que cada bloco continha duas réplicas de cada UA (I, BA e BB; quatro unidades de cada tipo por bloco, considerando-se que uma mesma área de mata contínua apresenta dois conjuntos de unidades amostrais).

Análise estatística - Os interiores e bordas de fragmentos e matas contínuas foram comparados quanto à riqueza de espécies (número de espécies, $S$ ) e à abundância de indivíduos (número total de indivíduos, $N$ ), através de análise de variância de dois fatores ("Two-Way" ANOVA, Sokal \& Rohlf 1995), em duas etapas. Primeiramente, comparou-se as áreas de controle (matas contínuas, > 900 ha) com as áreas de fragmento (25-100 ha), testando a interação das mesmas com os blocos amostrais, para verificar a existência de algum tipo de "efeito de área-core" (efeitos pronunciados sobre as comunidades biológicas devidos à redução no tamanho da área de recurso, Laurance et al. 1998a). Em segundo lugar, realizou-se a segunda etapa da análise, comparando-se as UA quanto à distância da borda e sua interação com os blocos.

Ressalta-se que a primeira etapa foi necessária para garantir a independência entre os fatores da segunda etapa, i.e., Interiores (I), Bordas externas (BA) e Bordas intermediárias (BB), parcelas estabelecidas para se quantificar a penetração do efeito de borda, ao longo do gradiente de distância em relação à divisa da floresta com a matriz.

Tecnicamente, a ANOVA não indica entre quais tratamentos estão as possíveis diferenças para as variáveis dependentes testadas. Portanto, para analisar a variação da riqueza entre as parcelas do "gradiente" (segunda etapa) utilizou-se a técnica de contrastes múltiplos ou "comparações planejadas" (Sokal \& Rohlf 1995), considerando-se, em primeiro lugar, Interiores vs. Bordas em geral $(\mathrm{BA}+\mathrm{BB}) \mathrm{e}$, posteriormente, BA vs. BB. Para a detecção da diferença de riqueza entre blocos, optou-se pelo teste de comparações múltiplas de Newman-Keuls, um teste baseado na distribuição estatística S - "studentized" (Newman 1939, Keuls 1952).

No entanto, nessa segunda etapa, a ANOVA somente pôde ser aplicada para a riqueza de espécies. $\mathrm{O}$ aporte estatístico empregado para as abundâncias totais (número de indivíduos em cada tratamento) foi uma adaptação não paramétrica da análise de variância de dois fatores ("TwoWay" ANOVA), conhecida como Scheirer-Ray-Hare (Scheirer et al. 1976), para número de amostras iguais. Este teste foi escolhido, pois as abundâncias não apresentaram homocedasticidade, nem mesmo com a realização de transformações matemáticas $\left(\log _{10}, \log _{e}\right.$, raiz quadrada, e ranqueamento).

A diferença básica entre Scheirer-Ray-Hare para ANOVA é que o primeiro considera os "quadrados médios" $(M S)$ referentes à variância total, incluindo a do erro padrão, na realização dos cálculos. Assim, a estatística da distribuição $F$, presente na ANOVA, encontra no similar não-paramétrico a sua equivalente, denominada $H_{m}$, cujo cálculo é efetuado da seguinte maneira: $H_{m}=\frac{R S S_{\text {tratamento }}}{R M S_{\text {total }}}$

onde: $H_{m}=$ parâmetro do cálculo de Scheirer-Ray-Hare; $R S S$ = soma dos quadrados nos tratamentos, calculada para postos ("ranks"); RMS = quadrado médio total. 
O cálculo de $M S_{\text {total }}$ é obtido pela soma total da soma dos quadrados (SS) dividida pelo número total de graus de liberdade. A distribuição $H$ é uma variante de $\chi^{2}$ e, portanto, a tabela utilizada para a obtenção dos valores das probabilidades é a mesma do $\chi^{2}$, com o número de graus de liberdade do tratamento testado. A utilização de Scheirer-RayHare é indicada, pois combina a computação prática e eficiente da ANOVA com a pequena demanda de pressupostos das análises não paramétricas, embora saiba-se que sua acurácia e robustez seja menor do que a ANOVA. Para todas as análises, o nível de significância adotado foi de $5 \%(\alpha=0,05)$.

\section{Resultados}

Riqueza e abundância de pteridófitas - Ao inventariar-se as pteridófitas das 36 parcelas foram encontrados 53 táxons entre as espécies e variedades identificadas, distribuídas nas áreas de maneira desigual (tabela 1): 46 espécies em interiores de floresta (I), sendo 13 exclusivas dessas áreas; 36 em bordas intermediárias (BB), sendo 4 exclusivas; e 27 em bordas mais externas (BA), das quais três foram exclusivas.

Tabela 1. Abundância total das espécies encontradas em unidades florestais, na região de Una, BA. IF = interiores de fragmento; $\mathrm{IC}=$ interiores de mata contínua; $\mathrm{BBF}=$ bordas intermediárias de fragmento; $\mathrm{BBC}=$ bordas intermediárias de mata contínua; $\mathrm{BAF}=$ bordas externas de fragmento $; \mathrm{BAC}=$ bordas externas de mata contínua.

Table 1. Total abundance of species found in sample units of the Una region. IF = interiors of fragments; IC $=$ interiors of continuous forest $\mathrm{BBF}=$ intermediate edges of fragments; $\mathrm{BBC}=$ intermediate edges of continuous forest; $\mathrm{BAF}=$ external edges of fragments; $\mathrm{BAC}=$ external edges of continuous forest.

\begin{tabular}{|c|c|c|c|c|c|c|c|}
\hline Família/Espécie & IF & IC & $\mathrm{BBF}$ & $\mathrm{BBC}$ & BAF & BAC & Total \\
\hline \multicolumn{8}{|l|}{ ASPLENIACEAE } \\
\hline Asplenium serratum $\mathrm{L}$. & 26 & 18 & 9 & 15 & 1 & 4 & 73 \\
\hline \multicolumn{8}{|l|}{ BLECHNACEAE } \\
\hline Blechnum brasiliense Desv. & 1 & & & & & & 1 \\
\hline \multicolumn{8}{|l|}{ CYATHEACEAE } \\
\hline Cyathea corcovadensis (Raddi) Domin & 71 & 82 & 32 & 43 & 18 & 24 & 270 \\
\hline Cyathea phalerata Mart. & 43 & 4 & 16 & & 40 & & 103 \\
\hline Cyathea abreviata Fernandes & 2 & 5 & & & & & 7 \\
\hline Cyathea pungens (Willd.) Domin & 6 & & & 1 & & & 7 \\
\hline \multicolumn{8}{|l|}{ DAVALLIACEAE } \\
\hline Nephrolepis spp.* & 14 & 3 & 27 & & 32 & 1 & 77 \\
\hline \multicolumn{8}{|l|}{ DENNSTAEDTIACEAE } \\
\hline Lindsaea lancea (L.) Bedd. var. lancea & 50 & 40 & 18 & 32 & 12 & 12 & 164 \\
\hline Lindsaea lancea (L.) Bedd. var. falcata (Dryand.) Rosenst. & 15 & 17 & 6 & 11 & & & 49 \\
\hline Lindsaea macrophylla Kaulf. & 11 & & 10 & 1 & 3 & & 25 \\
\hline Pteridium aquilinum (L.) var. arachnoideum (Kaulf.) Brade & & & & & 1 & & 1 \\
\hline Lindsaea guianensis (Aubl.) Dryand. & & 1 & & & & & 1 \\
\hline \multicolumn{8}{|l|}{ DRYOPTERIDACEAE } \\
\hline $\begin{array}{l}\text { Cyclodium heterodon (Schrad.) T. Moore } \\
\text { var. abbreviatum (C. Presl.) A.R. Sm. }\end{array}$ & 478 & 140 & 237 & 222 & 153 & 123 & 1353 \\
\hline Polybotrya cylindrica Kaulf. & 153 & 16 & 65 & 16 & 24 & 5 & 279 \\
\hline Cyclodium meniscioides (Willd.) C. Presl. var. meniscioides & 42 & 66 & & 17 & & 4 & 129 \\
\hline \multicolumn{8}{|l|}{ HYMENOPHYLLACEAE } \\
\hline Trichomanes pinnatum Hedw. & 21 & 23 & 7 & 64 & 3 & 12 & 130 \\
\hline Trichomanes elegans Rich. & 4 & 12 & 10 & 33 & & 4 & 63 \\
\hline Trichomanes polypodioides Raddi & 1 & 8 & & 8 & & & 17 \\
\hline Trichomanes pedicellatum Desv. & 2 & 1 & 1 & 6 & 1 & 2 & 13 \\
\hline Trichomanes crispum L. & 2 & & & & & & 2 \\
\hline \multicolumn{8}{|l|}{ LOMARIOPSIDACEAE } \\
\hline Lomagramma guianensis (Aubl.) Ching & 201 & 468 & 608 & 583 & 482 & 528 & 2870 \\
\hline Lomariopsis marginata (Schrad.) Kuhn & 86 & 128 & 79 & 124 & 30 & 87 & 534 \\
\hline Elaphoglossum pteropus C. Chr. & 3 & 26 & & 1 & & 1 & 31 \\
\hline Elaphoglossum sp. & & 9 & & & & & $\begin{array}{c}9 \\
\text { continuc }\end{array}$ \\
\hline
\end{tabular}


continuação

\begin{tabular}{|c|c|c|c|c|c|c|c|}
\hline Família/Espécie & IF & IC & $\mathrm{BBF}$ & $\mathrm{BBC}$ & BAF & $\mathrm{BAC}$ & Total \\
\hline \multicolumn{8}{|l|}{ LOMARIOPSIDACEAE } \\
\hline Elaphoglossum macrophyllum (Mett. ex Kuhn) Christ & 1 & 1 & 4 & & & & 6 \\
\hline Elaphoglossum consobrinum (Kunze) T. Moore & & 1 & & & & & 1 \\
\hline \multicolumn{8}{|l|}{ MARATTIACEAE } \\
\hline Danaea elliptica $\mathrm{Sm}$. & 2 & 5 & & 14 & 1 & 1 & 23 \\
\hline \multicolumn{8}{|l|}{ METAXYACEAE } \\
\hline Metaxya rostrata (Kunth) C. Presl. & 4 & & & & & & 4 \\
\hline \multicolumn{8}{|l|}{ POLYPODIACEAE } \\
\hline Polypodium meniscifolium Langsd. \& Fisch. & 8 & 18 & 1 & 1 & & 1 & 29 \\
\hline Microgramma vacciniifolia (Langsd. \& Fisch.) Copel. & & 1 & 3 & 2 & 1 & 4 & 11 \\
\hline Microgramma geminata (Schrad.) R.M. Tryon \& A.F. Tryon & 2 & 1 & 4 & 1 & & & 8 \\
\hline Microgramma lycopodioides (L.) Copel. & 1 & & 1 & 1 & & & 3 \\
\hline Campyloneurum repens (Aubl.) C. Presl. & 2 & & & & & & 2 \\
\hline Polypodium triseriale $\mathrm{Sw}$. & & & & & & 1 & 1 \\
\hline Pecluma ptilodon (Kunze) M.G. Price & 1 & & & & & & 1 \\
\hline Dicranoglossum desvauxii (Klotzsch) Proctor & 1 & & & & & & 1 \\
\hline Pecluma plumula (Humb. \& Bompl. ex Willd.) M.G. Price & & & 1 & & & & 1 \\
\hline Polypodium latipes Langsd. \& Fisch. & & & 1 & & & & 1 \\
\hline Polypodium polypodioides (L.) Watt. var. polypodioides & & & & 1 & & & 1 \\
\hline \multicolumn{8}{|l|}{ PTERIDACEAE } \\
\hline Adiantum dolosum Kunze & 2 & 1 & 3 & 8 & & 7 & 21 \\
\hline Adiantum lucidum (Cav.) Sw. & 1 & 10 & & 7 & & & 18 \\
\hline Pteris splendens Kaulf. & 1 & & 4 & & & & 5 \\
\hline \multicolumn{8}{|l|}{ SCHIZAEACEAE } \\
\hline Lygodium volubile $\mathrm{Sw}$. & 42 & 24 & 41 & 27 & 39 & 9 & 182 \\
\hline Schizaea elegans (Vahl.) Sm. & 1 & 3 & 3 & & & 1 & 8 \\
\hline Anemia phyllitidis (L.) Sw. & 3 & & 1 & & 3 & & 7 \\
\hline Schizaea fluminensis Miers ex J.W. Sturm & & 7 & & & & & 7 \\
\hline \multicolumn{8}{|l|}{ SELAGINELLACEAE } \\
\hline Selaginella producta Baker & 14 & 3 & 1 & 1 & 2 & 7 & 28 \\
\hline \multicolumn{8}{|l|}{ TECTARIACEAE } \\
\hline Triplophyllum funestum (Kunze) Holttum var. funestum & 130 & 50 & 18 & 18 & 23 & 6 & 245 \\
\hline \multicolumn{8}{|l|}{ THELYPTERIDACEAE } \\
\hline Thelypteris macrophylla (Kunze) C.V. Morton & & & & & 11 & & 11 \\
\hline Thelypteris longifolia (Desv.) R.M. Tryon & 2 & & & & & & 2 \\
\hline Thelypteris dentata (Forssk.) E.P. St. John & 1 & & & & & & 1 \\
\hline Thelypteris conspersa (Schrad.) A.R. Sm. & & & 1 & & & & 1 \\
\hline \multicolumn{8}{|l|}{ VITTARIACEAE } \\
\hline Vittaria lineata (L.) J. Sm. & 1 & & & & & & 1 \\
\hline Total & 1452 & 1192 & 1212 & 1258 & 880 & 844 & 6838 \\
\hline
\end{tabular}

* Nephrolepis spp. refere-se a duas espécies: N. pectinata (Willd.) Schott e N. rivularis (Vahl) Ching.

Todas as assembléias de espécies das unidades estudadas (I, BB e BA) estão distribuídas de acordo com a curva log-normal para a abundância das espécies (hipótese canônica de Preston, Preston 1948), segundo a qual "muitos processos devem agir em conjunto sobre a ecologia da comunidade estudada" (May 1975), existindo um maior número de espécies com abundância intermediária na comunidade, do que espécies raras ou dominantes. De acordo com o índice de diversidade $\lambda$, da distribuição log-normal (Kempton \& Taylor 1974), $\mathrm{BB}$ tende a apresentar uma comunidade mais diversa do que BA ( $\lambda=42,53$ e $\lambda=32,80$, respectivamente), mas, menos diversas do que os interiores $(\lambda=53,50)$. As comunidades de samambaias desses ambientes 
ajustam-se segundo o modelo de distribuição com elevada confiabilidade $\left(\chi_{B A}^{2}=4,11 ; p>0,75, \chi_{B B}^{2}=2,98\right.$; $p>0,95$ e $\left.\chi_{I}^{2}=4,55 ; p>0,75\right)$. Dessa forma, tais unidades podem ser consideradas áreas de diversidade relativamente alta, nas quais a maioria das espécies apresenta abundância intermediária e apenas algumas poucas são dominantes ou raras.

As ANOVA realizadas para detectar possíveis diferenças entre a riqueza e a abundância das unidades amostrais revelaram que a riqueza de espécies e a abundância total de indivíduos de pteridófitas não são influenciadas pelo tamanho do fragmento, ou seja, não é verificado nenhum tipo de "efeito de área-core" $\left(F_{1,30}=0,004 ; p=0,961\right.$, para riqueza e $F_{1,30}=0,123$; $p=0,728$, para abundância) (tabela 2, figura 2A, B). Entretanto, blocos amostrais comparados entre si apresentaram riquezas diferentes $\left(F_{2,30}=4,943\right.$; $p=0,014$ ) (tabela 2, figura 2C), quando são considerados os valores totais médios deste parâmetro. Este resultado refletiria a existência de heterogeneidade em macroescala, ou seja, com relação à paisagem, e indicaria que as áreas florestais presentes em diferentes blocos podem ser distintas com relação a características ambientais, não estudadas no presente trabalho. Nenhuma diferença foi encontrada com relação à abundância total entre blocos $\left(F_{2,30}=1,148 ; p=0,331\right.$, tabela 2, figura 2D).

A partir das diferenças detectadas para os blocos, verificou-se quais deles apresentavam riquezas de espécie distintas, através do teste de comparações múltiplas de Newman-Keuls. As florestas do bloco 2 apresentaram as assembléias mais ricas, em contraste com as do bloco 3, mais pobres (tabela 3, figura 2C). Provavelmente, esta diferença é ocasionada pela presença, no bloco 3, de áreas de mata contínua extremamente pobres em pteridófitas, como a Trilha do Príncipe (UA11), na qual foram encontradas apenas quatro espécies. Devido ao bloco 3 estar localizado mais a leste entre todos os três, grande parte do mesmo apresenta solos mais arenosos, oriundos da Formação Barreiras, localmente oligotrofizados (Leão \& Gouvêa 1971), o que representa uma limitação para a ocorrência de algumas espécies de pteridófitas que necessitam de substratos ricos em nutrientes minerais.

Uma vez que não foram detectadas diferenças de riqueza e abundância de pteridófitas entre matas contínuas e fragmentos pequenos, pôde-se analisar, independentemente do tamanho da área, os tratamentos I, BA e BB em relação aos blocos amostrais, a fim de verificar se existe efeito de borda e qual a sua distância de penetração floresta adentro.

A riqueza variou tanto entre as parcelas localizadas a diferentes distâncias da borda $\left(F_{2,30}=4,354\right.$; $p=0,023)$, quanto entre os blocos $\left(F_{2,30}=5,832\right.$; $p<0,001)$ (tabela 2). Ao se verificar entre quais blocos ocorre a diferença, confirmam-se as observações anteriores de que o bloco 2 é o que apresenta maior número de espécies de pteridófitas, enquanto o bloco 3 é o mais pobre de todos (Newman-Keuls, $p<0,01$ ).

A interação entre os fatores 1 e 2 da ANOVA (parcelas do gradiente de distância e blocos) não mostrou variações significativas da riqueza média

Tabela 2. Resumo das análises de variância de dois fatores usadas para testar os efeitos do tamanho das áreas de floresta (A) e a distância à linha de borda (B), sobre a riqueza de espécies e o número total de indivíduos de pteridófitas na região de Una, BA. Diferenças significativas são consideradas para $p$ valores $<0,05$.

Table 2. Summary of two-way ANOVA used to test the area-core effects (A) and the penetration of edge effect (B) on pterydophyte richness and abundance of the Una region. Significant differences are considered to $p$ values $<0,05$.

\begin{tabular}{|c|c|c|c|c|c|c|}
\hline & \multirow[t]{2}{*}{ Análise } & \multirow{2}{*}{$\begin{array}{l}\text { Graus de } \\
\text { liberdade }\end{array}$} & \multicolumn{2}{|c|}{$S$} & \multicolumn{2}{|c|}{$N$} \\
\hline & & & $F$ & $P$ & $F$ & $P$ \\
\hline \multirow{3}{*}{ (A) } & Área & 1 & 0,0046 & 0,9461 & 0,1232 & 0,7281 \\
\hline & Bloco & 2 & 4,9432 & 0,0139 & 1,1482 & 0,3308 \\
\hline & Interação & 2 & 1,1705 & 0,3240 & 2,9694 & 0,0666 \\
\hline \multirow{3}{*}{ (B) } & Distância & 2 & 4,3536 & 0,0230 & $* 2,1358$ & $* * 0,50<p<0,25$ \\
\hline & Bloco & 2 & 5,8317 & 0,0079 & $* 4,7345$ & $* * 0,50<p<0,25$ \\
\hline & Interação & 4 & 0,6131 & 0,6568 & $* 5,4384$ & $* * 0,25<p<0,10$ \\
\hline
\end{tabular}

* Proporções obtidas através da estatística $H$ (Scheirer et al. 1976). ** Probabilidades ( $p$ ) obtidas através da comparação com o valor crítico $\alpha=0,05$ de $\chi^{2}$. 

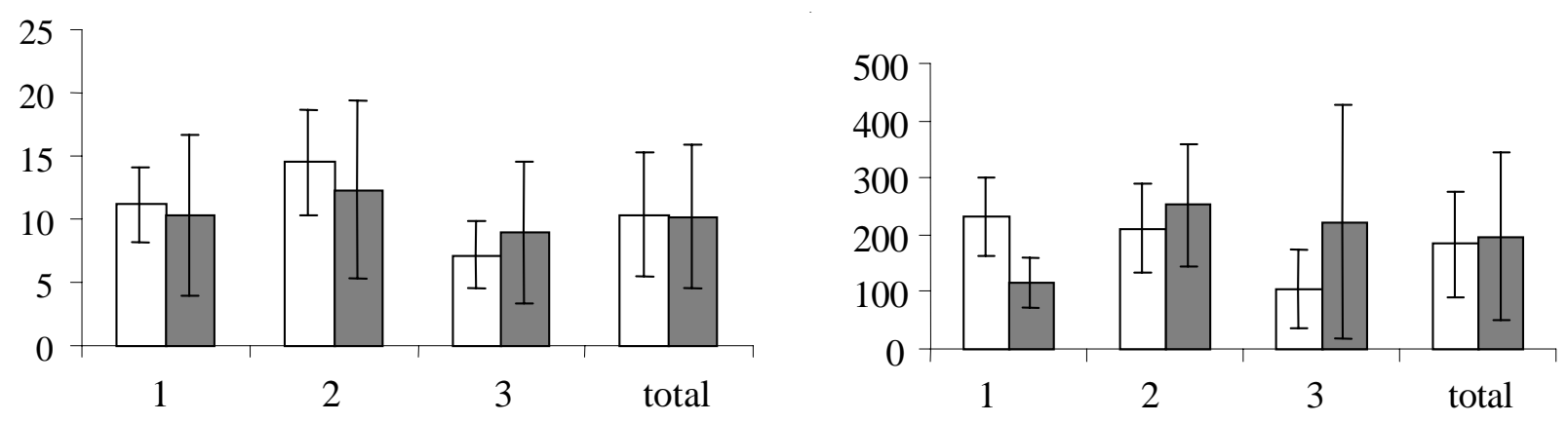

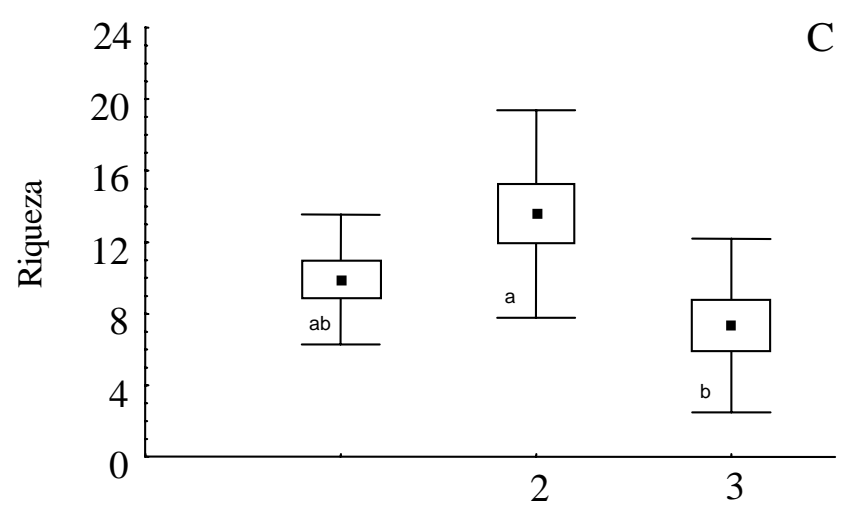

Blocos amostrais

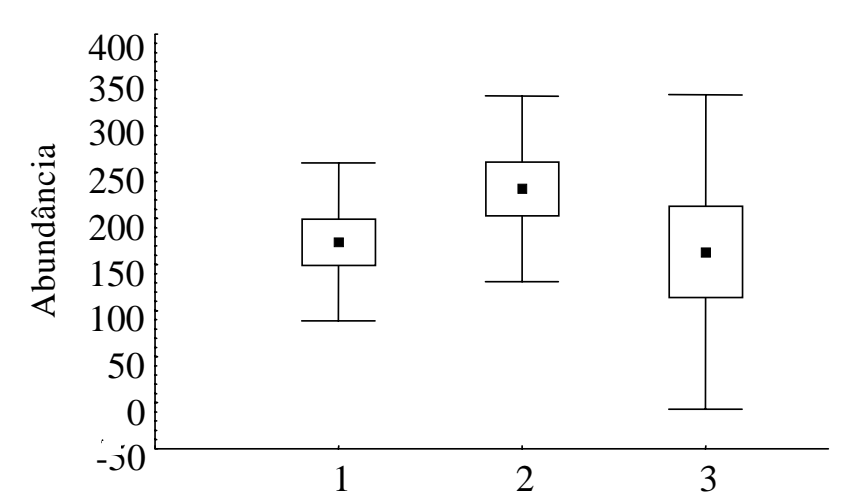

Blocos amostrais

Figura 2. Riqueza e abundância das áreas florestais contínuas e fragmentadas, em três blocos amostrais. Média e desvio padrão da riqueza (A) e da abundância (B) de controles e fragmentos [em ambos os casos, não foram detectadas diferenças significativas $\left(F_{1,30}=0,004 ; p=0,946\right.$ e $\left.\left.F_{1,30}=0,123 ; p=0,728\right)\right]$. Média e desvio padrão da riqueza (C) e abundância (D) totais entre os blocos amostrais, considerando-se o tamanho da área de floresta, como o primeiro fator da ANOVA. Diferenças são detectadas somente para a riqueza de espécies, entre os blocos 2 e $3\left(F_{2,30}=4,943 ; p=0,013\right)$. ( $\square=$ controles, $\square=$ fragmentos).

Figure 2. Pteridophyte communities species richness and abundance in continuous and fragmented forest on the three blocks. Mean and standard deviation of the species richness (A), and abundance (B) in forested areas. In both cases there's no significant differences $\left(F_{1,30}=0,004 ; p=0,946\right.$ e $\left.F_{1,30}=0,123 ; p=0,728\right)$. Mean and standard deviation of the total species richness (C), and abundance (D) in experimental blocks. In those cases forest areas size were considered the first ANOVA factor, and significant differences exist between blocks 2 and 3 to species richness $\left(F_{2,30}=4,943 ; p=0,013\right)$. $(\square=$ control, = fragments).

$\left(F_{4,30}=0,613 ; p=0,657\right.$; tabela 2$)$ e, assim, pode-se assumir que estes dois fatores são independentes na determinação da maior ou menor riqueza encontrada, contribuindo para corroborar a hipótese edáfica, anteriormente sugerida.

As abundâncias, por sua vez, não foram diferentes em nenhuma das instâncias quando testadas através de Scheirer-Ray-Hare $\left(H_{(2,33)}=4,7345 ; p>0,25 \mathrm{e}\right.$ $H_{(2,33)}=2,1358 ; p>0,25$, para o gradiente de distância e para blocos, respectivamente) (tabela 2).

Dentre as áreas do gradiente, os interiores florestais mostraram-se áreas totalmente distintas daquelas de borda quanto à riqueza de espécies (tabela 4), quando essas últimas foram consideradas em conjunto entre todos os blocos $\left(\mathrm{I} \times \mathrm{BA}+\mathrm{BB} ; F_{1,30}=6,981 ; p=0,014\right)$.

As bordas, entre si, também mostraram diferenças de riqueza $\left(F_{2,30}=4,354 ; p=0,023\right)$, sugerindo que as parcelas responsáveis pela diferença entre interiores e bordas são aquelas mais externas (BA), e não todas as parcelas de borda. Portanto, é possível que o efeito de borda influencie negativamente a riqueza de espécies de pteridófitas até uma distância de aproximadamente $20 \mathrm{~m}$ da linha de borda e, pelo contrário, pode-se verificar um incremento linear da riqueza à medida em que se avança em direção ao interior da floresta (figura 3A).

Em linhas gerais, a fragmentação de hábitats florestais em Una e o conseqüente aumento do número 
Tabela 3. Teste de comparações múltiplas (Newman-Keuls) para a riqueza de espécies entre os blocos amostrais, considerando-se o tamanho dos fragmentos (ANOVA Área $x$ Bloco; ver tabela 2-A). São consideradas diferenças significativas os $p$ valores $<0,05$.

Table 3. Multiple comparison test (Newman-Keuls) of the species richness among sample blocks considering the fragments area (ANOVA Area $\times$ Bloco; see table 2-A). Significant differences are considered to $p$ values $<0,05$.

\begin{tabular}{ccc}
\hline Riqueza média & Newman-Keuls & $p$ \\
\hline 9,92 & bloco $1 \times$ bloco 2 & 0,0765 \\
13,58 & bloco $1 \times$ bloco 3 & 0,2059 \\
7,33 & bloco $2 \times$ bloco 3 & 0,0106 \\
\hline
\end{tabular}

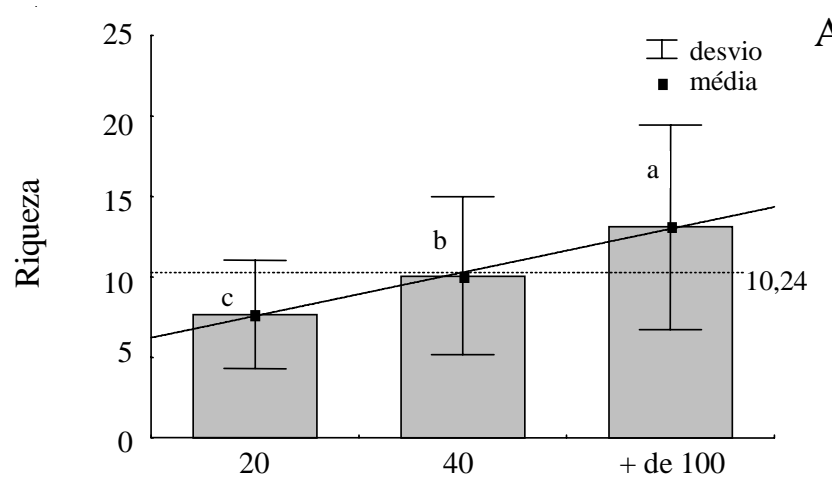

Distância à matriz (m)

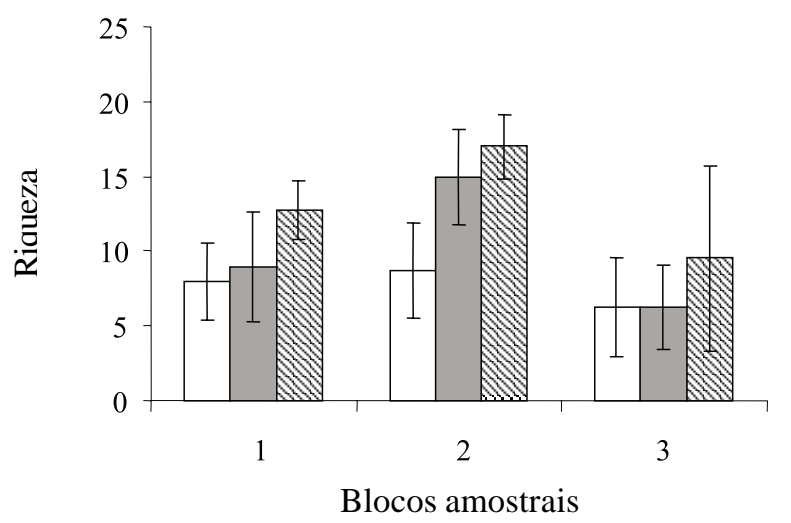

Figura 3. Variação da riqueza de pteridófitas da borda para o interior da floresta, para todos os blocos amostrais (A) [diferenças significativas são verificadas entre "a" e "b/c" $\left(F_{1,30}=6,981 ; p=0,014\right)$ e entre "b" e "c" $\left(F_{2,30}=4,354\right.$; $p=0,023)]$ e para cada um dos blocos (B). ( $\square=$ bordas A, = bordas $\mathrm{B}, \mathrm{k}=$ interiores).

Figure 3. Gradual improvement of pterydophytes species richness from edge to interior: (A) for all experimental blocks [there's differences between "a" and "b/c" $\left(\mathrm{F}_{1,30}=6,981\right.$; $p=0,014)$, and "b" and "c" $\left.\left(\mathrm{F}_{2,30}=4,354 ; p=0,023\right)\right]$; $(\mathrm{B})$ for each block. ( $\square=$ borders A, $\square=$ borders B, $=$ interiors $)$.
Tabela 4. Teste de comparações planejadas (contrastes múltiplos) para a riqueza de espécies entre o "gradiente" florestal (fator 1 da ANOVA; ver tabela 2-B).

Table 4. Planned comparisons test of the forest gradient species richness (factor 1 of the ANOVA; see table 2-B).

\begin{tabular}{lccc}
\hline Análise & $\begin{array}{c}\text { Graus de } \\
\text { liberdade }\end{array}$ & $F$ & $P$ \\
\hline Interiores $\times$ Bordas $(\mathrm{BA}+\mathrm{BB})$ & 1 & 6,9806 & 0,0135 \\
$\mathrm{BA} \times \mathrm{BB}$ & 2 & 4,3536 & 0,0230 \\
\hline
\end{tabular}

de bordas, pode levar a uma perda do número de espécies, em escala regional, o que é percebido a uma distância relativamente curta do limite do fragmento com a área aberta circundante. Pode-se notar que o estabelecimento deste aumento de espécies tende a ocorrer em todos os blocos (figura 3B), resultando no padrão geral encontrado.

Quanto à abundância média total, embora não tenha sido detectada nenhuma diferença significativa entre blocos ou entre as parcelas do gradiente, nota-se uma tênue tendência geral ao aumento do número de indivíduos conforme se adentra na floresta (figura 4).

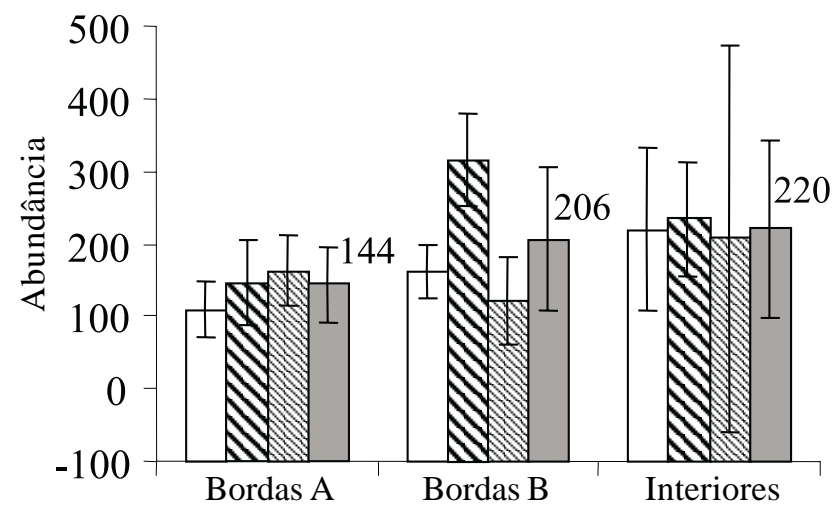

Figura 4. Variação da abundância total média de pteridófitas entre as áreas estudadas, em cada conjunto de unidades do gradiente de distância. ( $\square=$ bloco $1, \mathbb{N}=$ bloco 2 , 圈= bloco 3, = total).

Figure 4. Total abundance of the pteridophyte community among study areas, on each group of distance gradient. ( $\square=$ block $1, \mathbb{N}=$ block 2 , 国= block $3, \square=$ total $)$.

\section{Discussão}

Na região de Una, as áreas de floresta não apresentaram nenhum tipo de diferença quanto à 


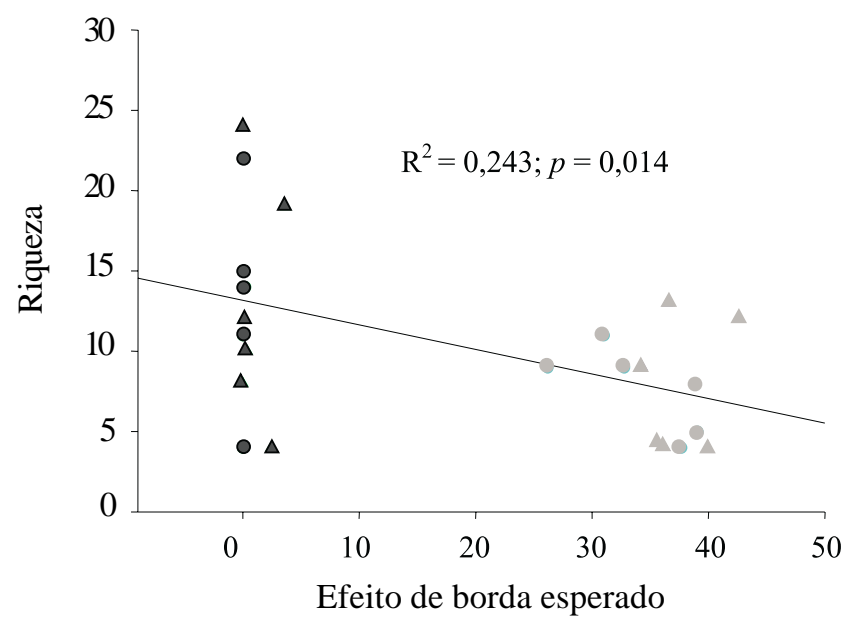

Figura 5. Relação linear entre a riqueza de pteridófitas e a intensidade do efeito de borda na região de Una, BA (regressão linear). Interiores de floresta tendem a apresentar um maior número de espécies quando comparados às bordas e esta diferença parece se pronunciar de forma abrupta entre os dois tipos de ambientes $\left(\mathrm{R}^{2}=0,243 ; p<0,05\right)(\bullet=$ interior de mata contínua ${ }^{\bullet}=$ borda de mata contínua $\boldsymbol{\Delta}=$ interior de fragmento $\Delta=$ borda de fragmento).

Figure 5. Linear relationship between pteridophytes species richness and the edge effect intensity, in Una region, State of Bahia, Brazil. Forest interiors should have more species when they are compared to edges. These difference is steep among those enviroments $\left(\mathrm{R}^{2}=0,243 ; p<0,05\right)(\bullet=$ interior of the continuous forest $\bullet=$ edge of the continuous forest $\boldsymbol{\Delta}=$ interior of fragments $\Delta=$ edge of fragments).

abundância, entre assembléias de pteridófitas de interiores e bordas, contrariando resultados obtidos por estudos realizados com outros grupos vegetais (e.g., Williams-Linera 1990, Matlack 1993, 1994).

Entretanto, nessas mesmas áreas florestais foi verificada uma forte diferença quanto à riqueza de espécies, pronunciada em duas instâncias: 1) quanto à disposição dos blocos amostrais (localização das áreas florestais), e 2) quanto à distância da borda, em relação ao interior da floresta.

O primeiro caso, no qual foi demonstrado que a riqueza de espécies de pteridófitas varia entre os blocos amostrais e, portanto, sobre a paisagem, sugere que as manchas de floresta podem estar sendo influenciadas por fatores não considerados no presente estudo. Isso porque tal situação ocorre independentemente do tamanho da mancha de floresta ou de sua proximidade da linha de borda, ou seja, a diferença encontrada se deve somente à disposição geográfica dos blocos em si e, provavelmente, os fatores responsáveis pela determinação dessa diferença devem estar relacionados a atributos geomorfológicos ou edáficos. Os blocos amostrais podem apresentar solos oriundos de formações distintas, assim como é verificado, de um modo geral, para a paisagem de Una (Leão \& Gouvêa 1971) e, originados por diferentes processos de intemperização, estes solos poderiam proporcionar uma alocação diferencial de micro e macronutrientes na escala da paisagem, interferindo na determinação da riqueza das pteridófitas. Parece plausível essa explicação, uma vez que o bloco 3, para o qual é conhecido o predomínio de solos oligotróficos (Leão \& Gouvêa 1971), mostrou-se o mais pobre em espécies de pteridófitas, mesmo sendo o bloco que detém a maior porção de área florestal contínua e conectada entre todos os três (RestaUna 2002). Assim, não devem ser as conexões entre manchas de floresta e a estrutura da mata propriamente dita os fatores responsáveis pela determinação da riqueza de espécies e, sim, as diferenças edáficas anteriormente mencionadas.

No segundo caso, considerando-se todas as 36 parcelas amostradas, em uma "mesoescala", demonstrou-se que a riqueza de espécies também é afetada pelo "efeito de borda". Efeitos de borda são resultantes da interação entre duas unidades de paisagem adjacentes, quando estas estão separadas por uma transição abrupta, que é a borda propriamente dita (Murcia 1995). No contexto deste trabalho, as zonas de transição são aquelas que ocorrem entre as florestas e as pastagens adjacentes e, embora a justaposição dos dois ecossistemas possa produzir efeitos em ambos, o interesse aqui é o efeito de borda sobre as manchas de mata remanescentes.

Neste estudo, foi constatado que os efeitos de borda influenciam negativamente a riqueza de pteridófitas, ocorrendo uma perda de espécies nos ambientes periféricos da floresta, quando estes são comparados aos interiores. No entanto, verifica-se diminuição efetiva de riqueza apenas junto às parcelas $\mathrm{BA}$ (primeiros $20 \mathrm{~m}$ ), uma vez que as parcelas intermediárias BB $(40$ m) já apresentam riqueza diferente da encontrada nas parcelas BA (tabela 4). Isto sugere que as pteridófitas devem estar respondendo a fatores ambientais de ação local e com penetração relativamente curta fragmento adentro que, por sua vez, podem ser intensificados pela formação de novas bordas nas florestas fragmentadas, resultando num processo sinérgico. Entre esses fatores, estão variáveis abióticas, como temperatura, umidade relativa do ar, umidade do solo e intensidade luminosa, que, nos ecossitemas florestais, formam um gradiente ambiental com tendência ao desaparecimento após os primeiros $50 \mathrm{~m}$ no interior da mata (Kapos 1989, 
MacDougall \& Kellman 1992, Matlack 1993).

Segundo Matlack (1994), a extensão da "zona de borda" (região afetada pelas alterações abióticas) é um fator crítico para a existência de um hábitat de interior em fragmentos florestais. Em Una, o efeito de borda é mais pronunciado principalmente dentro dos primeiros $20 \mathrm{~m}$ das bordas de floresta e os interiores de floresta correspondem a ambientes relativamente homogêneos quanto às espécies estudadas, sendo pouco influenciados pela penetração dos efeitos de borda. De acordo com um modelo empírico de efeito de borda proposto por Malcolm (2001) - que considera a proporção de matriz circundante em relação a um determinado ponto dentro de um fragmento, independentemente de sua área total - a pressão ecológica exercida pela matriz sobre a determinação do efeito de borda, para a comunidade de pteridófitas, num dado ponto, pouco interessa para explicar a penetração dos efeitos de borda sobre os interiores florestais, que praticamente não são afetados, pois a distância desta penetração tende a ser muito curta. Por outro lado, as parcelas BA formam um conjunto no qual todas as unidades são afetadas intensamente pelo efeito de borda e mesmo efeitos mais sutis não devem ser detectados a distâncias maiores. Uma relação linear negativa descreve o comportamento da riqueza de espécies com a intensificação do efeito de borda anteriormente mencionado (figura 5), explicando $24,31 \%$ da variação encontrada para este parâmetro $(p=0,014)$. Assim, os fatores estruturais da borda, como seus aspectos micro-climáticos (ventos, temperatura, umidade relativa, evapotranspiração) e, provavelmente, o nível de perturbação, seriam importantes fontes de variação da riqueza de espécies, corroborando os resultados verificados por Fox et al. (1997) para florestas da Austrália.

Se existe uma correlação entre tais fatores e o estabelecimento/colonização das pteridófitas, então pode-se supor que a menor riqueza detectada nas bordas BA ocorre, em última análise, devido à existência de diferenças entre as tolerâncias fisiológicas das espécies, em resposta às alterações ambientais. Resultados algo similares foram obtidos por Bernabe et al. (1999) para o desenvolvimento de esporófitos de samambaias arbóreas. Com relação a esta questão, é importante salientar que o próprio ciclo de vida das pteridófitas impõe um obstáculo ecológico à ocorrência das espécies: o esporófito sempre é limitado a hábitats propícios ao desenvolvimento dos gametófitos, que são organismos muito sensíveis às mudanças micro-climáticas bruscas, principalmente nas espécies tropicais (Page 1979, Kornás 1985, Ranal 1995). Uma situação ambiental que não beneficia alguma das gerações acaba sendo limitante para o sucesso da outra, ainda que as duas fases sejam independentes, do ponto de vista biológico. Implicitamente, as pteridófitas são vegetais que raramente conseguem sobreviver em condições ambientais extremas (Page 1979), como aquelas supostamente presentes nas bordas mais externas dos fragmentos florestais (no caso, BA), onde atuariam o dessecamento intenso e duradouro, dado o aumento de temperatura e a diminuição da umidade relativa.

Embora seja conhecido que a orientação e a fisionomia das bordas sejam aspectos importantes na modulação da intensidade de grande parte dos efeitos abióticos (Murcia 1995, Esseen \& Renhorn 1998), apenas os dados de fisionomia puderam ser acessados para a região (Pardini 2001). Sabe-se que a comunidade de espécies arbóreas determina a arquitetura e as condições micro-climáticas da floresta e, portanto, as mudanças na dinâmica dessa comunidade podem influenciar o fluxo de dispersão/ colonização de outras espécies florestais, ou mesmo, outros processos ecológicos (Murcia 1995, Laurance et al. 1998a, b). Em florestas tropicais, o clima externo à mata, mais severo que o do interior, é tamponado pelo dossel denso, mas tal tamponamento desaparece próximo à borda da floresta, onde a queda de folhas aumenta substancialmente (Lovejoy et al. 1986, Williams-Linera 1990), podendo acarretar alta mortalidade das plantas sensíveis ao dessecamento. Se tal situação efetivamente ocorre também nas florestas de Una, segundo indica Dixo (2001), é plausível de se supor que isso contribui para o estabelecimento de um suposto estresse hídrico nas pteridófitas adaptadas para habitar ambientes sombreados como os interiores florestais.

Embora Didham \& Lawton (1999) tenham encontrado um efeito de área sobre o micro-clima e sobre a estrutura da floresta, o mesmo não pode ser afirmado para a região de Una, nem mesmo para parâmetros biológicos como a riqueza de espécies. Esses mesmos autores ressaltam que seus resultados parecem ser uma resposta ao tipo de borda em questão: se está isolada ou em um contexto em que há muitas bordas próximas umas às outras. Em Una, a quantidade de bordas próximas e de áreas relativamente abertas não parece influenciar a riqueza de pteridófitas.

Pode-se concluir que, enquanto muitos fatores abióticos variam como uma função simples da distância da borda de floresta, através da formação de um gradiente, segundo diversos dados presentes na literatura (Kapos 1989, Matlack 1994, Kapos et al. 1997), sistemas bióticos, tais como a riqueza e a abundância 
de espécies, podem não responder da mesma forma, o que já foi atestado por outros autores (Murcia 1995, Didhan \& Lawton 1999).

Assim, sejam as variações no micro-clima da floresta ou a estrutura do estrato arbóreo (proporcionando uma maior incidência de ventos fortes próximos às bordas) as condicionantes do estabelecimento das pteridófitas nas florestas da região, o fato é que a fragmentação causa uma pronunciada perda de espécies nos remanescentes de Una, e um dos fatores mais importantes dessa diminuição em fragmentos e áreas contínuas de mata são os efeitos de borda. Em locais onde a criação e ampliação de bordas dificilmente pode ser evitada, tal como em Una, deveriam ser implementados programas de plantio de mudas de árvores nativas de crescimento rápido, em frente às bordas, o que poderia reduzir a penetração de um suposto gradiente abiótico e, conseqüentemente, amenizar a perda de espécies de pteridófitas sensíveis.

Agradecimentos - Ao Projeto RestaUna (Probio/Pronabio/ MMA - CNPq/Bird-GEF) e a todos os seus pesquisadores, pelo suporte logístico e apoio incondicional para a realização deste estudo; ao Ibama, na pessoa do Sr. Saturnino Souza, por permitir o acesso à Rebio; aos Dr. Renata Pardini, Jean Paul Metzger e Rodrigo Leão Moura, pelo auxílio nas análises; ao Instituto de Pesquisas Ecológicas (IPE) pelo uso do programa Statistca 5.0; à Capes pela bolsa de estudo concedida ao primeiro autor.

\section{Referências bibliográficas}

ALGER, K. 1997. Dinâmica do uso da terra no entorno da Reserva Biológica de Una. In Alternativas econômicas para o desenvolvimento da região de Una, Bahia (K. Alger, ed.). Instituto de Estudos Sócio-Econômicos da Bahia, Ilhéus.

BERNABE, N., WILLIAMS-LINERA, G. \& PALACIOS-RIOS, M. 1999. Tree ferns in the interior and at the edge of a Mexican cloud forest remnant: spore germination and sporophyte survival and establishment. Biotropica 31:83-88.

BROWN, K.S. 1991. Conservation of Neotropical enviroments: insects as indicators. In Conservation of insects and their natural enviroments (N.M. Collins \& J.A. Thomas, eds.). R.E.S. Symposium 15. Academic Press, London, p.350-404.

CI. 2002. Conservation International. Hot-spots. http:// www.conservation.org/Hotspots. (acesso em 20/01/2002).

DIDHAN, R.K. \& LAWTON, J.H. 1999. Edge structure determines the magnitude of changes in microclimate and vegetation structure in tropical forest fragments. Biotropica 31:17-30.
DIXO, M. 2001. Efeitos da fragmentação da floresta sobre a comunidade de sapos e lagartos de serapilheira no Sul da Bahia. Dissertação de mestrado, Universidade de São Paulo, São Paulo.

ESSEEN, P. \& RENHORN, K. 1998. Edge effects on an epiphytic lichen in fragmented forests. Conservation Biology 12:1307-1317.

FORMAN, R.T.T. \& GODRON, M. 1986. Landscape Ecology. Wiley \& Sons, New York.

FOX, B.J., TAYLOR, J.E., FOX, M.D. \& WILLIAMS, C. 1997. Vegetation changes across edges of rainforest remnants. Biological Conservation 82:1-13.

FRANKLIN, J.F. 1993. Preserving biodiversity: species, ecosystems, or landscapes? Ecological Applications 3:202-205.

GOUVÊA, J.B.S. 1969. Contribuição à geomorfologia do sul da Bahia. Área dos baixos cursos dos rios Pardo e Jequitinhonha. Comunicação Técnica do Centro de Pesquisas do Cacau / Comissão Executiva de Planejamento da Lavoura Cacaueira, Itabuna, v.35, p.1-11.

GOUVÊA, J.B.S., SILVA, L.A.M. \& HORI, M. 1976. Fitogeografia. In Diagnóstico sócio-econômico da região cacaueira. Recursos Florestais. Ceplac, Instituto Interamericano de Ciências Agrícolas, Ilhéus, v.7, p.1-17.

HAFFER, J. 1974. Avian speciation in tropical South America. Publications of the Nuttall Ornithological Club 14:1-390.

KAPOS, V. 1989. Effects of isolation on the water status of forest patches in the Brazilian Amazon. Journal of Tropical Ecology 5:173-185.

KAPOS, V., WANDELLI, E., CAMARGO, J.L. \& GANADE, G. 1997. Edge-related changes in enviromental and plants responses due to forest fragmentation in central Amazonia. In Tropical forest remnants: ecology, management, and conservation of fragmented communities (W.F. Laurance \& R.O. Bierregaard, eds.). The University of Chicago Press, Chicago, p.33-44.

KEMPTON, R.A. \& TAYLOR, L.R. 1974. Log-series and lognormal parameters as diversity determinants for the Lepidoptera. Journal of Animal Ecology 43:381-399.

KEULS, M. 1952. The use of the "studentized range" in connection with analysis of variance. Euphytica 1:112-122.

KOEPPEN, W. 1948. Climatologia. Con un estudio de los climas de la tierra. Versión directa de Pedro R. Hendrichs Pérez. Fondo de Cultura Económica, Ciudad de México.

KORNÁS, J. 1985. Adaptative strategies of African pteridophytes to extreme environments. In Biology of Pteridophytes. (A.F. Dyer \& C.N. Page, eds.). The Royal Society of Edinburgh, Edinburgh, p. 391-396.

LAURANCE, W.F. 1991. Edge effects in tropical forest fragments: applications of a model for the design of nature reserves. Biological Conservation 57:205-219.

LAURANCE, W.F., FERREIRA, L.V., MERONA, J.M.R. \& LAURANCE, S.G. 1998a. Rain forest fragmentation and the dynamics of Amazonian tree communities. Ecology 79:2032-2040. 
LAURANCE, W.F., FERREIRA, L.V., MERONA, J.M.R., LAURANCE, S.G., HUTCHINGS, R.W.\&LOVEJOY, T.E. 1998b. Effects of forest fragmentation on recruitment patterns in Amazonian tree communities. Conservation Biology 12:460-464.

LEÃO, A.C. \& GOUVÊA, J.B.S. 1971. Uso atual das terras da região cacaueira do Estado da Bahia - Fôlhas Itabuna, Una, Potiraguá, Mascote e Canavieiras. BoletimTécnico Cepec / Ceplac 8: 5-22.

LOVEJOY, T.E., BIERREGAARD, R.O., RYLANDS, A.B., MALCOLM, J.R., QUINTELA, C.E., HARPER, R.H., BROWN, K.S., POWELL, A.H., SCHUBART, O.R. \& HAYS, M.B. 1986. Edge and other effects of isolation on Amazonian forest fragments. In Conservation biology (M.E. Soulé, ed.). The science of scarcity and diversity. Sinauer Associates, Sunderland, Massachusetts, p.237-256.

MACDOUGALL, A. \& KELLMAN, M. 1992. The understorey light regime and patterns of tree seedlings in tropical riparian forest patches. Journal of Biogeography 19:667-675.

MALCOLM, J.R. 1994. Edge effects in Central Amazonian forest fragments. Ecology 75:2438-2445.

MALCOLM, J.R. 2001. Extending models of edge effects to diverse landscape configuration. In Lessons from Amazonia: The ecology and conservation of a fragmented forest (R.O. Bierregaard, C. Gascon, T.E. Lovejoy \& A. Santos, eds.). Yale University Press, New Haven.

MATLACK, G.R. 1993. Microenvironment variation within and among forest edge sites in the eastern United States. Biological Conservation 66:185-194.

MATLACK, G.R. 1994. Vegetation dynamics of the forest edge - trends in space and successional time. Journal of Ecology 82:113-123.

MAY, R.M. 1975. Patterns of species abundance and diversity. In Ecology and evolution of communities (M.L. Cody \& J.M. Diamond, eds.). Cambridge University Press, Cambridge, p.81-120.

METZGER, J.P. 1999. Estrutura da paisagem e fragmentação: análise bibliográfica. Anais da Academia Brasileira de Ciências 71:445-463.

MORI, S.A., BOOM, B.M., CARVALHO, A.M. \& SANTOS, T. 1983. Southern Bahian moist forests. The Botanical Review 49:155-232.

MURCIA, C. 1995. Edge effects in fragmented forests: implications for conservation. Trends in Ecology and Evolution 10:58-62.

NEWMAN, D. 1939. The distribution of range in samples from a normal population, expressed in terms of an independent estimate of standard deviation. Biometrika 31:20-30.

NICHOL, J.E. 1994. An examination of tropical rain forest microclimate using GIS modeling. Global Ecology and Biogeography Letters 4:69-78.
PAGE, C.N. 1979. Experimental aspects of fern Ecology. In The Experimental Biology of Ferns. (A.F. Dyer, ed.). Academic Press, London, p.551-589.

PARDINI, R. 2001. Pequenos mamíferos e a fragmentação da Mata Atlântica de Una, sul da Bahia: padrões, processos e conservação. Tese de doutorado, Universidade de São Paulo, São Paulo.

PRANCE, G.T. 1982. A review of the phytogeographic evidences for pleistocene climate changes in the neotropics. Annals of the Missouri Botanic Garden 69:594-624.

PRESTON, F.W. 1948. The commonness, and rarity, of species. Ecology 29:254-283.

RANAL, M. A. 1995. Estabelecimento de pteridófitas em mata mesófila semidecídua do Estado de São Paulo. 1. Caracterização Climática do Ambiente. Anais da Academia brasileira de Ciências 67:351-367.

RESTAUNA. 2002. Projeto RestaUna: Remanescentes de Floresta da Região de Una, Sul da Bahia. http://www. restauna.org.br. (acesso em 12/04/2002).

RYLANDS, A.B. 1982. The behaviour and ecology of three species of marmosets and tamarins (Callitrichidae, Primates) in Brazil. PhD thesis, University of Cambridge, Cambridge.

SCHEIRER, C.J., RAY, W.S. \& HARE, N. 1976. The analysis of ranked data derived from completely randomized factorial designs. Biometrics 32:429-434.

SEI. 1998. Anuário Estatístico - Bahia. Edição da Superintendência de Estudos Econômicos e Sociais da Bahia (SEI), Salvador, v.12.

SOKAL, R.R. \& ROHLF, F.J. 1995. Biometry. $3^{\text {rd }}$ ed., W.H. Freeman, New York.

THOMAS, W., CARVALHO, A.M.V., AMORIM, A.M.A., GARRISON, J. \& ARBELÁEZ, A.L. 1998. Plant endemism in two forests in southern Bahia, Brazil. Biodiversity and Conservation 7:311-322.

TURNER, I.M. 1996. Species loss in fragments of tropical rain forest: a review of the evidence. Journal of Applied Ecology 33:200-209.

WIENS, J.A., STENSETH, N.C., VAN HORNE, B. \& IMS, R.A. 1993. Ecological mechanisms and landscape ecology. Oikos 66:369-380.

WILLIAMS-LINERA, G. 1990. Vegetation structure and environmental conditions of forest edges in Panama. Journal of Ecology 78:356-373.

WILLIAMS-LINERA, G., DOMÍNGUEZ-GASTELÚ, V. \& GARCÍA-ZURITA, M.E. 1997. Microenvironment and floristics of different edges in a fragmented tropical rainforest. Conservation Biology 12:1091-1102.

WILLSON, M.F. \& CROME, F.H.J. 1989. Patterns of seed rain at the edge of a tropical Queensland rain forest. Journal of Tropical Ecology 5:301-308. 
